\title{
ASENTAMIENTOS INFORMALES, INUNDACIONES Y VULNERABILIDAD FÍSICO- ESPACIAL DE LA VIVIENDA Y EL ENTORNO INMEDIATO Caso de estudio Arroyo La Esmeralda en el Distrito de Barranquilla, Colombia.
}

\author{
Autor: Myrna Inés Arévalo Carmona \\ Universidad en la que se desarrolla la investigación: Universidad de Chile \\ Director de Tesis: Ricardo Tapia Zarricueta \\ Email: myrnaarevalo@mail.uniatlantico.edu.co
}

\section{RESUMEN}

En este artículo se presenta un enfoque metodológico para identificar variables de análisis de la vulnerabilidad, desde la dimensión físico espacial, y permitir una mejor comprensión del estado en el sector Suroccidental del Distrito de Barranquilla, como un aporte a la metodología incorporada para el Plan de Ordenamiento Territorial. Muchas de las características propias del lugar que configuran condiciones físicas y aportan a la vulnerabilidad social no fueron incluidas en esta, por lo tanto, se conoce menos acerca de su comportamiento en un sector no homogéneo de origen informal. A partir de la operacionalización del concepto de vulnerabilidad se identifican variables y características de la vivienda y el entorno inmediato que inducen a una descripción desde la micro escala, vinculadas con las prácticas de habitar y la forma de apropiación inherentes del lugar.

Palabras Clave: Vulnerabilidad Físico-espacial, inundaciones, Barranquilla y asentamientos informales.

\begin{abstract}
This article presents a methodological approach to identify variables of vulnerability analysis, from the spatialphysical dimension, and to allow a better understanding of the state in the Southwestern sector of the Barranquilla District, as a contribution to the incorporated methodology for the POT (Zonning Plan). Many of the characteristics of the place that physical-spatial conditions and contribute to social vulnerability were not included in this, therefore, less is known about their behavior in a non-homogeneous sector of informal origin. The operationalization of the vulnerability concept identifies variables and housing characteristics and the environment provided that induce a description from the micro-scale, linked to the practices of living and the form of appropriation Inherent in the place.
\end{abstract}

Key words: physical-spatial vulnerability, floods, Barranquilla and informal settlements. 


\section{INTRODUCCIÓN}

\subsection{El estudio de vulnerabilidad a inundaciones en el Sector Suroccidental del Distrito de Barranquilla, Colombia.}

En la metodología de evaluación de la vulnerabilidad a inundaciones realizada en Barranquilla, durante el proceso de incorporación de la Gestión de Riesgo de Desastre ${ }^{1}$ y revisión del Plan de Ordenamiento Territorial del Distrito en 2014, se identificaron características físico-espaciales de la vivienda y de su entorno inmediato que escasamente logran describir la exposición influyente en su estado.

Las condiciones evaluadas responden a un enfoque cuantitativo que se limita a medir cuatro (4) indicadores comprendidos desde una visión a escala de ciudad. Un (1) indicador refiere la exposición de elementos urbanos para perfilar los daños materiales o económicos de características urbanas afectadas o influyentes y los otros tres (3) describen características materiales y estructurales de la vivienda como el estado, altura (número de pisos arquitectónicos) de la construcción y tipo de estructura (ver gráfica 1) (Alcaldía Distrital de Barranquilla, 2014).

\begin{tabular}{|c|c|c|c|}
\hline $\begin{array}{l}\text { VARIABLES DEL } \\
\text { ESTUDIO POR EL }\end{array}$ & \multicolumn{3}{|c|}{$\begin{array}{l}\text { CARACTERISTICAS FISICO-ESPACIALES DEL ESTUDIO POR EL } \\
\text { INSTRUMENTO DE PLANEACIÓN }\end{array}$} \\
\hline INSTRUMENTO & VIVIENDA & ENTORNO INMEDIATO & SECTOR/CIUDAD \\
\hline FACTOR DE & & Usos de suelo expuestos & Vías \\
\hline EXPOSICIÓN & & Espacio público expuesto. & Acueducto /alcantarillado \\
\hline FRAGILIDAD & $\begin{array}{l}\text { Estado de deterioro de la } \\
\text { edificación }\end{array}$ & & \\
\hline $\begin{array}{l}\text { SOCIAL Y FALTA DE } \\
\text { RESILIENCIA }\end{array}$ & $\begin{array}{c}\text { Características particulares } \\
\text { de la vivienda (materiales y } \\
\text { niveles) }\end{array}$ & & \\
\hline
\end{tabular}

Ese enfoque desconoce los diferentes niveles de vulnerabilidad que probablemente se generaron por el desarrollo urbano de la ciudad. Para el caso de Barranquilla, las características urbanas y de la vivienda son el resultado de una ocupación espacial, sin nociones de Ordenamiento Territorial, que no vinculó el sistema natural de desagüe del terreno. Como consecuencia, un total de 12.377 viviendas se vieron afectadas por las inundaciones ocurridas en la cuenca occidental del Distrito entre 2008 y 2012 (ver gráfico 2) (Alcaldía Distrital de Barranquilla, 2014).

Dentro de ese esquema, el Sector Suroccidente de la ciudad se configura sobre la cuenca, desde los años cincuenta (Alcaldía Distrital de Barranquilla, 2014), mediante un desarrollo urbano de origen informal bajo las condiciones socioeconómicas de una comunidad de bajos recursos (Torres, 2009). Aunque la evaluación tuvo en cuenta características de la vivienda y el entorno para categorizarlo como un sector con alto nivel de riesgo a inundaciones, se conoce poco acerca de la modalidad de exposición y el estado de vulnerabilidad que enfrentan los habitantes.

Hasta el momento no se conocen los resultados de esa evaluación, solo se mostraron zonas de amenaza de inundación con alturas de 1.5 a $3.0 \mathrm{~m}$ en quince tramos de arroyos menores de la cuenca (Alcaldía Distrital de Barranquilla, 2014). Las zonas identificadas en el mapa de amenazas manifiestan una homogeneidad en el fenómeno, por lo tanto, las acciones de mitigación son estandarizadas. Las principales medidas estructurales son la canalización en concreto reforzado del cauce natural del arroyo para evitar el derrumbe de las orillas y las afectaciones a la vivienda. Sin embargo, no se permite controlar los efectos negativos de la inundación en el modo de vida de los habitantes quienes asumen medidas de mitigación con recursos propios y de forma

${ }^{1}$ La incorporación al instrumento se sujeta a las directrices de la Ley 1523 de 2012. 
experimental, pues los diseños son limitados al caudal y desconocen factores como el drenaje del terreno aledaño y el modo de implantación de las viviendas.

La vulnerabilidad total de un lugar es resultado de características intrínsecas del entorno construido, sistema natural y el sistema social, si los lugares son desiguales (Cutter et. al., 2003; Cutter et. al. 2008) deben establecer la evaluación del estado a partir de la interacción entre los sistemas que lo configuran y el reconocimiento de las características propias (Arévalo, 2016).

En términos de vulnerabilidad físico-espacial, el sector tiene probablemente niveles de vulnerabilidad más altos que otros sectores de la ciudad pues, debido a su origen, enfrenta la localización de asentamientos sobre las rondas hídricas de los arroyos, la inestabilidad estructural y constructiva de la vivienda (Torres, 2009) y las relaciones espaciales conflictivas entre la configuración morfológica del barrio, la tipología de la vivienda y el sistema de desagüe pluvial. Diferentes situaciones se presentan que no pueden ser analizadas de forma estándar y cuantitativa.
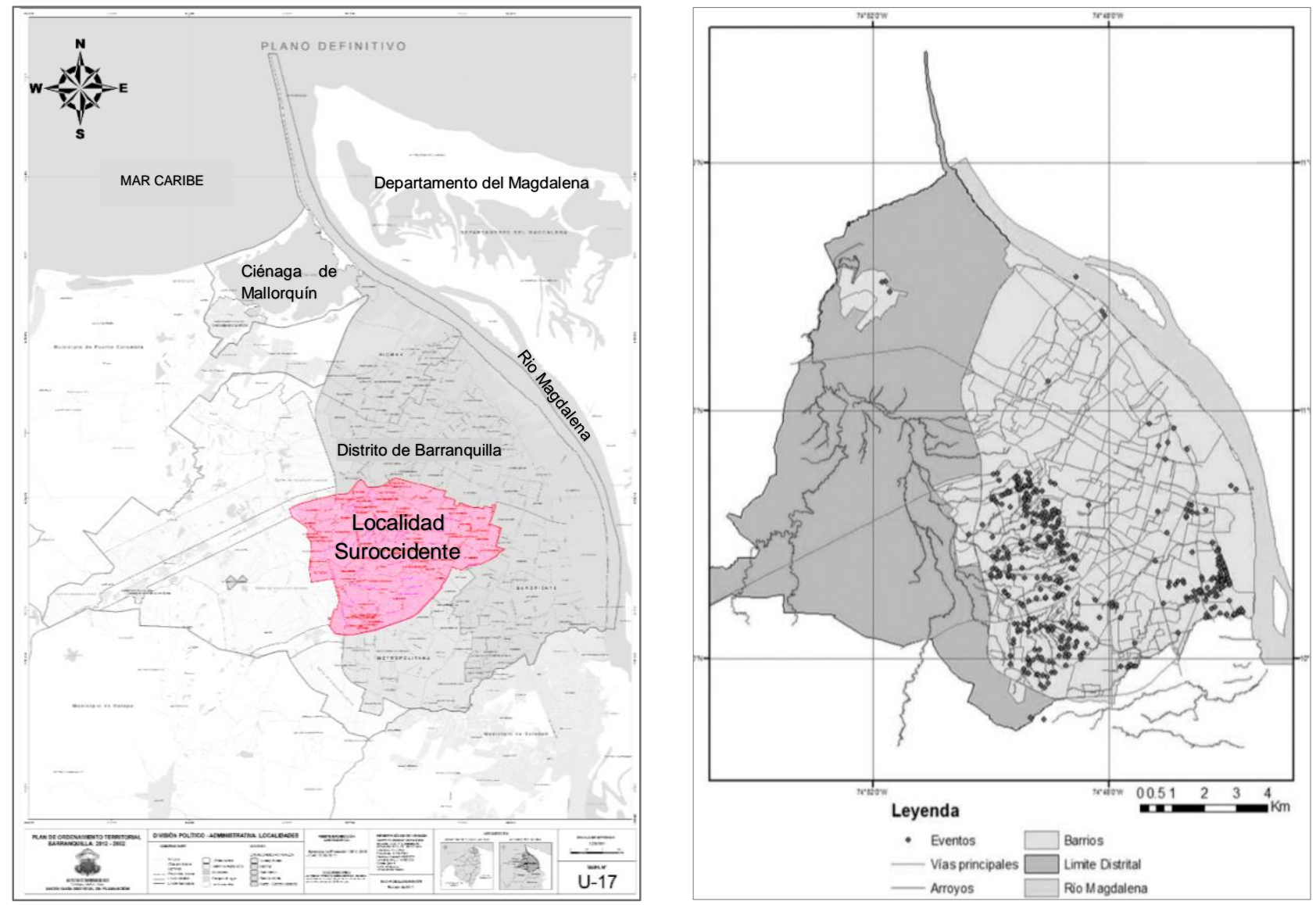

Gráfica 2. Localización y población vulnerable a inundaciones 2008-2012 en el Sector Suroccidental del Distrito de Barranquilla. (Alcaldía Distrital de Barranquilla, 2014)

Este artículo, basado en la investigación para la tesis de maestría en Hábitat Residencial entre el 2014 y 2015 , pretende reconocer las características físico-espaciales de la vivienda y el entorno inmediato que configuran su estado de vulnerabilidad dado su origen informal estos asentamientos y que no fueron vinculadas a la metodología desarrollada en la incorporación de la Gestión de Riesgo de Desastre al instrumento de planificación. Se trabaja con el supuesto que, al analizar las relaciones físico-espaciales entre la vivienda y su entorno inmediato, desde la escala micro como un proceso de hábitat dinámico, se permite conocer cómo están expuestos la vivienda y los elementos urbanos y cómo se configura su estado de vulnerabilidad.

Para ello se pregunta ¿Cuáles serían las variables para integrar en la metodología de estudio de la vulnerabilidad a inundaciones de Barranquilla que permitirían una mejor evaluación de los riesgos, de acuerdo las características 
físico-espaciales y medioambientales de los asentamientos expuestos en el sector suroccidental de la ciudad, tomando como referencia la problemática de la población localizada en la zona de inundación del arroyo La Esmeralda en el barrio Ciudad Modesto?

En los alcances de la investigación, se formuló como objetivo general: Proponer un conjunto de variables para el análisis de vulnerabilidad de lugar que describan las características físico-espaciales de la vivienda y el entorno inmediato de la comunidad localizada sobre zona inundable del arroyo La Esmeralda en el sector Suroccidental de Barranquilla, que deban ser integradas a la metodología de estudio de la vulnerabilidad a inundaciones en la ciudad". Los objetivos específicos buscaron 1) Identificar las características urbanas del entorno inmediato en las zonas de inundación. 2) Identificar las características físico-espaciales de la vivienda en los asentamientos sobre la zona de inundación. 3) Vincular la percepción del riesgo de la comunidad sobre la relevancia de los elementos físicos expuestos y susceptibles a la inundación en los instrumentos de estudio de la vulnerabilidad y 4) Sistematizar un conjunto de variables acorde a la realidad de la vulnerabilidad físico-espacial del fenómeno y a las características intrínsecas del lugar desde el concepto de hábitat residencial.

\section{NOCIONES CONCEPTUALES PARA LA OPERACIONALIZACIÓN DEL CONCEPTO}

\subsection{La Vulnerabilidad percibida desde los conceptos de Hábitat Residencial, Pobreza y Asentamientos Informales.}

Interpretar el concepto de vulnerabilidad, implica establecer su relación con la amenaza, la exposición y el papel que juega en la construcción del riesgo. A partir de esa premisa, diversas y abstractas han sido las perspectivas que asume el concepto (Cutter et. al, 2008; Mohamed, 2009) que no logran abordar la cotidianidad de la comunidad (Romero y Mendoça, 2012) y las relaciones derivadas de la interacción entre los diferentes componentes de los sistemas que lo conforman (INVI, 2011). En el enfoque del riesgo, manejado por Romero y Mendoça, hacen énfasis en que la relación entre sociedad (estructura social y acciones humanas) y el sistema natural o ecológico construyen el riesgo acorde a los diferentes niveles de vulnerabilidad de las personas expuestas y la complejidad de la amenaza, haciendo referencia a tiempos y espacios de exposición (2012).

Frecuentemente, la vulnerabilidad se entiende como una aptitud o disposición del sistema social a ser probablemente afectado por un desastre (Wilches-Chaux, 1993; Vargas, 2002; Chardón, 2010; Alcaldía Distrital de Barranquilla, 2014) como la prefiguración de un escenario futuro analizado como un estado estático (Cutter et. al., 2008) sujeto a la presencia de elementos expuestos a amenazas de un entorno físico, que es afrontada por la capacidad de resistir de la comunidad.

La finalidad de medir la vulnerabilidad es definir estrategias de mitigación efectivas que permitan ajustar las características de los elementos que la configuran (Birkmann, 2006). La medición es basada en indicadores de enfoque cuantitativo dada la necesidad de lectura y manejo para la toma decisiones (Mustafa, 2011). Básicamente, se mide en términos de daños y pérdidas materiales y humanas (Chardón, 2010), mientras que el conocimiento sobre las características de los lugares que configuran su estado de vulnerabilidad es escaso (Cutter et. al., 2008). Poco se registra sobre los trastornos en el modo de habitar de la comunidad (Chardón, 2010).

El enfoque del hábitat, como enfoque integrador (Chardón, 2010), aporta una perspectiva para interpretar la complejidad de los procesos de la vida cotidiana que configuran la vulnerabilidad. La comprensión del concepto desde la realidad del fenómeno, como un proceso de hábitat, indica reconocer su carácter dinámico y multidimensional (Cutter et. al., 2003; Cutter et. al., 2008; Chardón, 2010) resultado de procesos de ocupación del territorio, la configuración de lugares y las características de la estructura social (INVI, 2011).

El Modelo de Vulnerabilidad en lugares de riesgo (Hazards-of-places Model of Vulnerability) (Cutter et. al., 2003) y el Modelo de Resiliencia a Desastres del Lugar (Disaster Resilence of Place Model - DROP) (Cutter et. al., 2008) desarrollado por el Instituto de Investigación de Riesgos y Vulnerabilidad (Hazards and Vulnerability Research Institute - HVRI) de la Universidad del Sur de California, definen que la vulnerabilidad es una característica inherente del lugar establecida por la interacción entre del entorno construido, sistema natural y sistema social. La calidad de esas relaciones, tornan sensible a los elementos expuestos por las condiciones de 
seguridad que se configuraron en el momento de adaptación y apropiación del lugar, pero principalmente por las cualidades intrínsecas del sistema social, su capacidad de responder ante el desastre y su resiliencia. Otros factores exógenos, tales como las medidas de mitigación desde los entes administrativos territoriales y la economía, influyen en la forma como se manejan las condiciones de seguridad (Cutter et. al., 2003; Cutter et. al., 2008).

Ello sugiere, que la vulnerabilidad se genera desde escalas territoriales micro influenciada por las escalas macro, pues su distribución espacial no es homogénea (Lindley et. al, 2011), las características físicas y sociales en un territorio son diversas, se genera impactos diferentes para algunos sectores de la población (niños, personas de la tercera edad, personas con condiciones especiales o grupos étnicos) (Ka'zmierczak \& Cavan, 2011) de acuerdo con las desigualdades derivadas de la ocupación de lugares desiguales (Cutter et. al., 2003).

Basado en esos modelos, para este artículo se asumió que "la vulnerabilidad es el resultado de la relación disfuncional en la interacción de los elementos expuestos" (Arévalo, 2016:35). Esa relación circunscribe un estado influyente que configura la calidad de la interacción entre las dimensiones del hábitat (físico-espacial, socio-cultural y político-económica) y, además, entre escalas territoriales (INVI, 2011). En los modelos del HVRI, la sensibilidad de elementos expuestos, sean receptores sociales o físicos, puede convertirse en un potenciador del riesgo.

En las ciudades colombianas, la configuración de la vulnerabilidad se vio influenciada por patrones informales de desarrollo territorial. La localización de asentamientos humanos en zonas no seguras para habitar (sectores periféricos, baldíos y con presencia de amenazas naturales) (Wilches-Chaux, 1993; Naranjo, 2004; Sánchez, 2007) corresponde a los problemas de acceso al suelo urbano para la población con bajos ingresos y a la insuficiencia de las acciones del estado para afrontarlo (Torres, 2009).

En la construcción del lugar, el sistema social define un ámbito dinámico definido por procesos sociales cambiantes que interactúan con factores exógenos y el espacio físico junto con las posibilidades de intervención connota un ámbito estático como una base físico-espacial (locación y la ubicación) caótica (Lombard, 2015). En asentamientos vulnerables de origen informal se producen situaciones de exposición, a la vez, que las condiciones socioeconómicas de la población potencian características de la exposición (Arévalo, 2016). Las condiciones para habitar se vuelven inseguras cuando las características del lugar no son adecuadas y requiere mayores recursos para adaptarse a él (Pérez, 2014). Es entonces cuando los diferentes niveles de vulnerabilidad se producen. Según Romero \& Mendoça, se presentan diferentes grados por cada persona dependiendo de la capacidad de adaptación de cada individuo (2012). Las limitaciones de acceso a los recursos y las privaciones para la realización de necesidades humanas apoyan la reproducción de espacios vulnerables, afectando el bienestar humano (Pérez, 2014). Generan procesos enmarcado por situaciones que fluctúan constantemente y cuyas posibilidades de superación del estado son limitadas.

La pobreza es una sumatoria de situaciones, refiere un sistema de accesos inadecuados a un nivel de calidad de vida. En entornos vulnerables se convierten factores de estrés y reproduce otros esquemas de vulnerabilidad pues el manejo simultaneo de situaciones diversas junto con la privación de las necesidades y las limitaciones de acceso a recursos conllevan a la trampa de la pobreza (Pérez, 2014).

Finalmente, se entiende que la vulnerabilidad es un proceso social soportado en una base territorial que fomenta y delimita las dinámicas de la comunidad (Cutter et. al., 2003). Las características espaciales que componen el entorno construido y natural vinculan acciones que influyen en la construcción de la vulnerabilidad social y, a su vez, influencia la configuración del aspecto físico. Una mayor descripción surge del análisis de estos aspectos físicos y permite establecer las condiciones vinculadas desde la forma de ocupación y habitar del lugar.

\subsection{Inundaciones en el modelo de ocupación de los asentamientos informales.}

Las inundaciones son consideradas amenazas variables, a pesar de su origen meteorológico, su intensidad depende de la duración, frecuencia de lluvias, condiciones del suelo, topografía y sistema del drenaje natural (Banco Mundial, 2012; Ka'zmierczak \& Cavan, 2011). Cuando el sistema de drenaje no alberga la cantidad de agua ocupa los terrenos aledaños, sus efectos son negativos por la acción antrópica al modificar el funcionamiento del drenaje del terreno (Banco Mundial, 2012). La medida de la amenaza por inundación impacta 
de forma diferencial a las personas conforme el nivel que alcanza, en una altura de $0.1 \mathrm{~m}$ afecta muebles, enseres y electrodomésticos, más de $0.1 \mathrm{~m}$ o mayores profundidades se afecta la estructura del inmueble, junto con la velocidad del agua la permanencia de las personas puede estar impedida (Ka'zmierczak \& Cavan, 2011). Entonces la familia requiere hacer inversiones en reparación o reposición acorde con la frecuencia de la inundación. Los efectos en otras escalas urbanas también se presentan por inconvenientes en el funcionamiento de las actividades incluso impidiendo la movilidad (Ávila, 2015).

\section{METOdología DE LA INVESTIGACIÓN}

Los estudios sobre la medición de la vulnerabilidad a inundaciones en el distrito de Barranquilla, ha sido escasos. Hasta el momento solo se conoce aspectos de la evaluación desarrollada durante el proceso de incorporación al instrumento de planificación del Distrito. Nuevos estudios desde enfoques actualizados son necesarios para aportar al conocimiento del fenómeno y evitar vacíos en la comprobación empírica. La investigación que se presenta en este artículo fue desarrollada bajo la modalidad de Actividad Equivalente a Tesis (AFE) para optar al título de Magister en Hábitat Residencial en la Universidad de Chile en 2016. En ella, se aplicó una investigación tipo exploratoria para detectar elementos del fenómeno que aporten una descripción apropiada y completa.

\subsection{Métodos}

La estrategia de investigación se desarrolló a partir de la operacionalización del concepto de vulnerabilidad para deducir las características urbanas del entorno inmediato y físico-espaciales de la vivienda que influencian y vinculan la vulnerabilidad social. En primer lugar, se delimitaron las dimensiones del concepto con base en la de una descripción conceptual de la vulnerabilidad total del lugar (Cutter et. al., 2003) elaborado por el Instituto de Investigación de Riesgos y Vulnerabilidad, que fue comprendido desde los conceptos de hábitat residencial (INVI, 2011), pobreza (Pérez, 2014), asentamientos informales (Lombard, 2015) e inundaciones (Ka'zmierczak \& Cavan, 2011). Para contar con una base robusta de características para seleccionar en la segunda etapa, se realizó el análisis de casos similares en Great Mánchester - Reino Unido y Valle de Aburrá (Medellín) - Colombia. Los casos fueron escogidos por la riqueza o variedad de características de la vivienda y el entorno inmediato identificadas en la dimensión físico espacial y la vinculación con un enfoque sistemático y escalar para analizar la vulnerabilidad a inundaciones. De este paso se obtuvo un conjunto de 17 características, definidas desde la comunidad científica, que describen la exposición y la influencia de la dimensión físico-espacial (ver gráfica 3). Seguidamente se seleccionaron las características correspondientes con tres criterios:

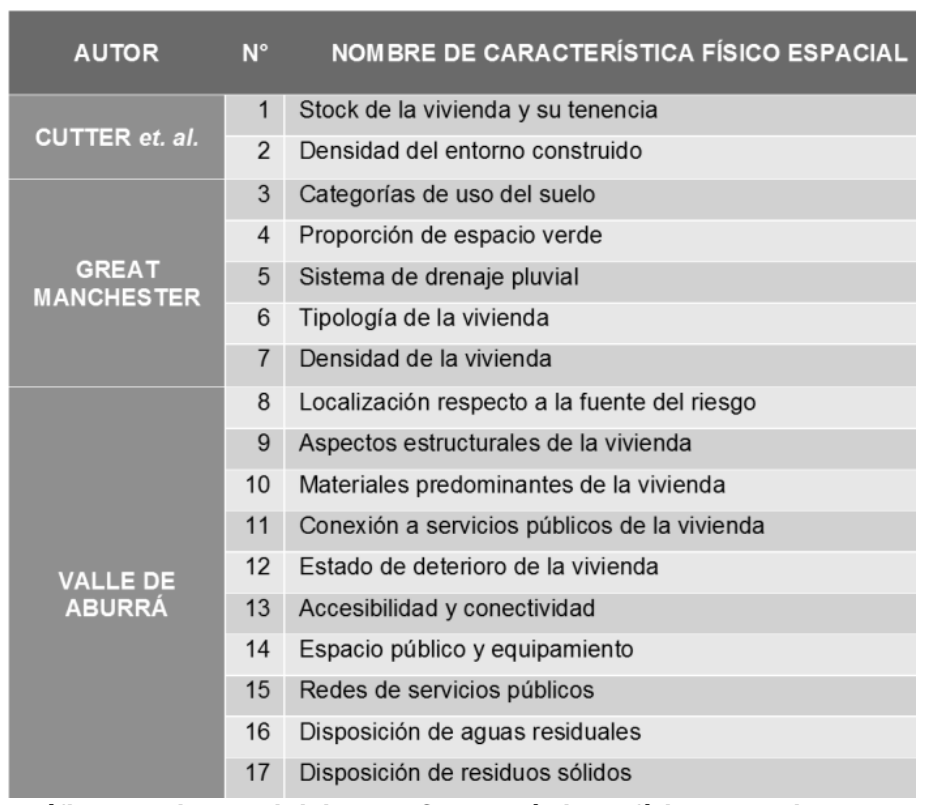

Gráfica 3. Listado inicial de Características físico-espaciales consideradas en el estudio de la vulnerabilidad según marco conceptual y estudio de casos.

Elaboración propia con base en Cutter et. al., 2003; Escuela de Hábitat, 2009; Ka’zmierczak \& Cavan, 2011. 
1) características que no fueron consideradas en la metodología de estudio de la vulnerabilidad a inundaciones del Distrito, se descartaron aquellas que ya estaban vinculadas en el modelo empleado en el caso de estudio.

2) características relevantes para la operacionalización del concepto consideradas con base en las dimensiones del concepto identificadas.

3) características que sean pertinentes con el contexto, para este último criterio se aplicaron dos instrumentos: expediente urbano y encuesta grupal. El conjunto final de diez (10) características se definieron gracias a este criterio.

El objeto del expediente urbano fue verificar en el caso de estudio del arroyo La Esmeralda, a través de observación directa, la presencia y manifestación de las características reconocidas en los dos primeros criterios de selección. Con el instrumento se pudo identificar la presencia de las características en el sector para ser analizadas en procesos de evaluación del Distrito. Además, demostró la existencia de otras que no estaban reconocidas desde la operacionalización del concepto. La ventaja del expediente es permitir una observación desde diferentes escalas (vivienda, entorno inmediato, sector y ciudad) y estudias las características asociadas a la morfología urbana del sector y la tipología de vivienda.

La confiabilidad y validez de los resultados se pusieron a prueba con una entrevista grupal a los habitantes en el caso de estudio. La indagación buscaba la opinión acerca de prácticas de habitar, experiencias durante período de lluvias e inundaciones ocurridas antes y después de la canalización, afectaciones de las inundaciones ocurridas a las viviendas y en el entorno, conocimiento de información acerca de las estrategias de respuesta durante la inundación y participación en el proceso de evaluación de la vulnerabilidad contratado por la Alcaldía de Barranquilla.

Como resultado, se obtuvieron cuatro (4) variables sistematizadas a través del concepto del Hábitat Residencial desarrollado por el Instituto de la Vivienda de la Universidad de Chile (INVI, 2011), las características obtenidas se analizaron y se compararon con las empleadas en por el instrumento de planificación del Distrito para la obtención de elementos en común con las dimensiones del concepto asumido en la investigación.

\subsection{Caso del estudio}

La metodología requirió del análisis en un caso de estudio de las características encontradas inicialmente, este se localizó en las manzanas con nivel de amenaza alta de inundación (1.5 y $3.0 \mathrm{~m}$ de altura) del arroyo la Esmeralda del barrio Ciudad Modesto (Ver gráfica 4). El área delimitada en el plano U-11 "Amenazas de Inundación" del Plan de Ordenamiento Territorial, corresponde a una zona principalmente residencial con estratificación socioeconómica baja. El arroyo pertenece a la cuenca Occidental enmarcada por la ladera del mismo nombre, catalogado como arroyo menor dentro de una red de afluentes y arroyos que desaguan en el arroyo León (arroyo mayor), la Ciénaga de Mallorquín hasta llegar al Mar Caribe. Aun sin ser un caso representativo del sector, pues en el suroccidente las quince áreas reconocidas con amenaza de inundación presentan el mismo nivel, es un caso con características propias correspondientes con el interés de la investigación como una trama urbana discontinua, un sistema de drenaje no resuelto y diferentes patrones de implantación o localización de la vivienda en la fuente de riesgo; todas ellas acorde con el origen del asentamiento.
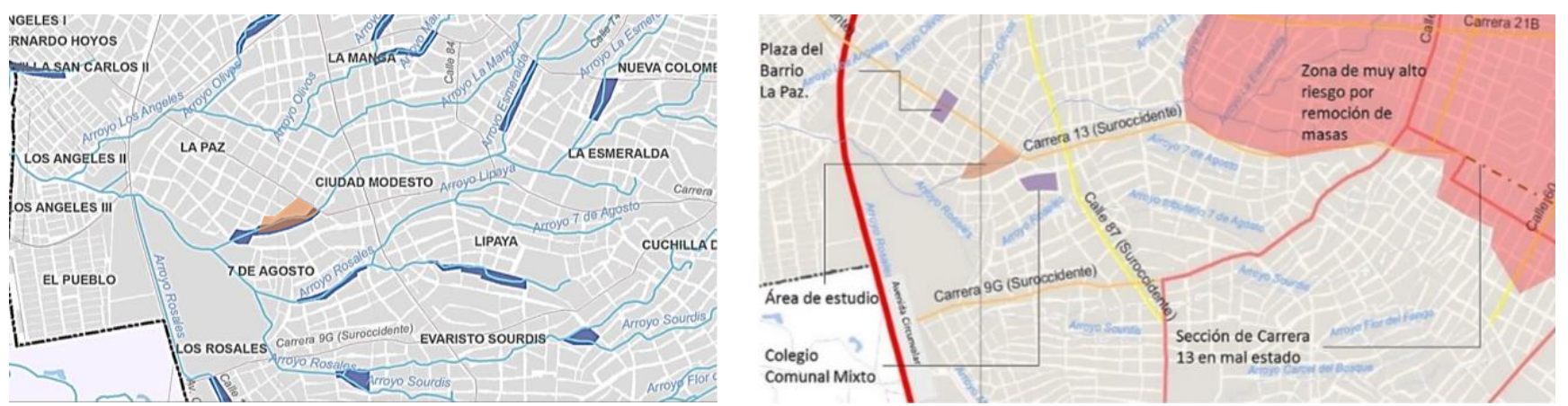

Gráfica 4. Localización y referencias del caso de estudio en el Sector.

Elaboración propia con base en Plan de Ordenamiento Territorial, planos U-11 y U12. 
Sobre el sistema de drenaje, cabe destacar que el Distrito no cuenta con alcantarillado pluvial, por lo tanto, en el sector las escorrentías se dirigen por la calle hacia el arroyo y pueden ingresar a la vivienda dependiendo de la localización, las medidas de mitigación asumidas por la comunidad o la administración, la topografía con pendientes del $5 \%$ y la morfología urbana espontánea.

\section{DIMENSIONES DEL CONCEPTO DE VULNERABILIDAD.}

Las dimensiones del concepto admiten el reconocimiento de las características urbanas y arquitectónicas que describen del estado de vulnerabilidad en el caso de estudio desde la escala de vivienda, entorno urbano y sector. A partir de esos aspectos, para el manejo del concepto en la investigación se reconocen dimensiones que permiten su comprensión. Estas son:

- Multidimensionalidad

- Relación escalar

- Dualidad entre elementos dinámicos y estáticos

- Disfuncionalidad.

En primera instancia, el aspecto multidimensional de la vulnerabilidad se manifiesta a partir de la influencia por el proceso sistémico del hábitat. Mientras los efectos en la construcción del lugar son delimitados de forma endógena algunos, también factores exógenos del territorio son influyentes (Cutter et. al., 2008). Las dimensiones físico-espacial y socioeconómica del lugar condicionan el entorno físico y natural (Arévalo, 2016) a través de patrones de exposición derivados de la forma y posibilidades de adaptación (Escuela de Hábitat, 2009). Estas son acciones delimitas por los factores económicos, psicológicos y subjetivos de la comunidad (Pérez, 2014). Por su parte, la dimensión política y económica manifiesta con los factores económicos del territorio (Cutter et. al., 2008), los tratamientos urbanos en el ordenamiento del territorio y las estrategias de mitigación en la gestión del riesgo.

En la dimensión físico-espacial, las dinámicas del hábitat presentan relaciones entre las escalas territoriales, es decir, que las características de la vivienda en el entorno inmediato y a su vez la configuración física de este, establece las relaciones con la morfología urbana y el sistema de drenaje del sector y la ciudad (Ka'zmierczak \& Cavan, 2011). Las escalas micro se apoyan en los atributos urbanos de escalas superiores que ofrecen posibilidades para el momento de enfrentar la amenaza, puesto que la capacidad de respuesta se constituye a partir del conocimiento la estructura urbana con rutas de evacuación, puntos seguros de encuentro y refugio (Ka'zmierczak \& Cavan, 2011). Los daños ocasionados a los elementos de la estructura urbana pueden debilitar la resiliencia de la comunidad (Cutter et. al., 2008; Ka'zmierczak \& Cavan, 2011).

Dentro de la escala micro, que para este artículo se entiende como vivienda y entorno inmediato, se plantea la dualidad entre elementos estáticos y dinámicos (Lombard, 2015), con el fin de comprender las dinámicas sociales que condicionan los patrones de exposición (dinámico/estático), las condiciones de esa exposición derivadas del proceso de adaptación que delimitan y potencian la vulnerabilidad social (Estático/Dinámico) y la interacción a diferentes escalas de los atributos del territorio con elementos urbanos y arquitectónicos expuestos que definen una vulnerabilidad física (Estático/exposición) (Arévalo, 2016).

El reconocimiento de la calidad de las relaciones entre los elementos expuestos (entorno construido, sistema natural y sistema social) refiere relaciones sistémicas conflictivas en el hábitat. En ese sentido, esas relaciones se presentan de modo disfuncional influyendo principalmente en las condiciones de la exposición (Arévalo, 2016). Las prácticas de habitar, la localización en zonas de amenazas (exposición), la forma de adaptación al sistema natural (Escuela de Hábitat, 2009) y los factores de estrés adyacentes construyen el panorama en el que se convive con el riesgo (Pérez, 2014). Enmarcan otra dimensión del concepto entendida como disfuncionalidad.

La calidad de las interacciones de los elementos expuestos, la escala en la que influye y el tipo de disfuncionalidad que se presenta es un aporte para entender la vulnerabilidad. En esta investigación, la exposición trasciende el enfoque habitual, correspondiente a la localización en una zona de amenaza, y se entiende como una condición físico-espacial dependiente de la correspondencia entre localización, las dinámicas territoriales en distintas escalas y el comportamiento de la inundación. Referencia las relaciones disfuncionales originadas por la forma de adaptación y las prácticas de habitar que incrementan o condicionan la vulnerabilidad social. A partir de ese 
precepto la selección de características para el análisis en este artículo es delimitada a aquellas que referencian esas dos disfuncionalidades.

\section{CARACTERÍSTICAS FÍSICO-ESPACIALES PROPUESTAS PARA MEDIR LA VULNERABILIDAD EN BARRANQUILLA}

La selección aplicada arrojó un conjunto de diez (10) características urbanas y arquitectónicas que se proponen como resultado de la investigación (ver gráfica 5). Un número equitativo referencia características de la vivienda y características urbanas que incrementan la exposición y limitan la respuesta. Al aplicar el segundo criterio de selección de las características se tuvo en cuenta la relevancia desde el enfoque conceptual y el interés de la investigación, por lo tanto, solo se identificaron aquellas que pueden describir la disfuncionalidad por apropiación del lugar y las prácticas de habitar, pues permiten comprender cómo es la exposición y cuáles características físico-espaciales logran influir de modo negativo.

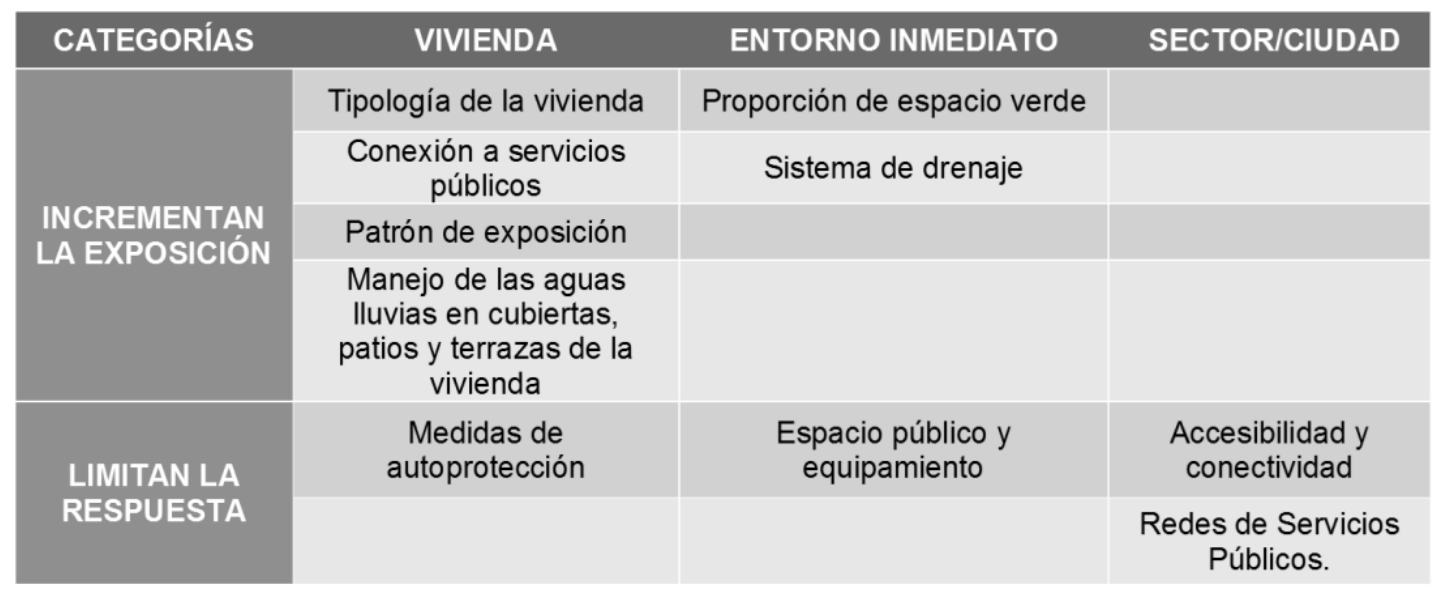

Gráfica 5. Conjunto de características físico-espaciales de la vivienda y el entorno propuestas para el estudio de la vulnerabilidad a inundaciones.

Elaboración Propia

A continuación, se presenta una definición de cada una indicando fuente, dimensión del concepto de vulnerabilidad que refiere, incidencia en la vulnerabilidad y manifestación en el caso de estudio.

\subsection{Definición de las características urbanas a escala de sector/ciudad propuestas}

\section{Accesibilidad y conectividad}

En el momento de la inundación la reacción se condiciona por las rutas de evacuación y acceso a zonas de resguardo cercanas o las disponibles en la ciudad. Sin embargo, su efectividad depende de la existencia, conocimiento y apropiación de la información correspondiente por parte de la comunidad. La Escuela de Hábitat en el Valle de Aburrá definió esta característica como una condición urbana que influye en la vulnerabilidad por la forma de adaptación (2009). El proceso de urbanización informal admite una trama vial discontinua que dificulta la evacuación efectiva en momentos de mayores inundaciones.

La localización del sector suroccidental del Distrito, en el margen izquierdo de la ladera occidental y la presencia de suelos de suelos geológicamente inestables (arcilla expansiva) que acotan el riesgo de remoción de masas, dificulta que la trama vial se conecte con el resto de la ciudad, reduciendo las posibilidades de evacuación a la vía circunvalar (vía perimetral). (Alcaldía Distrital de Barranquilla, 2014)

\section{Redes de Servicio Públicos}

Según la Escuela de Hábitat, la presencia de redes de servicios públicos posibilita la conexión de la vivienda, disminuyendo acometidas ilegales sin garantías de control y calidad. Esta situación se origina en el proceso de adaptación e incide en factores de estrés que hacen a la comunidad más vulnerable, pues no tiene acceso a servicios de acueducto, alcantarillado, energía eléctrica y gas domiciliario para resolver necesidades básicas (2009) 
De acuerdo con el Plan de Ordenamiento Territorial, el Distrito tiene una cobertura del $97 \%$ en Acueducto y total en Energía y Alcantarillado (Alcaldía de Barranquilla, 2014). Indica que existen posibilidades de controlar conexiones inseguras que afectarían al momento de retomar la cotidianidad después de la inundación.

\subsection{Definición de las características urbanas a escala de entorno inmediato propuestas}

\section{Proporción de áreas verdes y permeables}

La presencia zonas permeables en el entorno facilita la absorción rápida de las escorrentías y puede evitar la acumulación de agua en la malla vial, la proporción de ellos es el factor urbano del sector que incide en la vulnerabilidad. De esa forma, las áreas de jardín, parques, vías sin pavimentar, zonas impermeabilizadas e incluso lotes sin construcción o intervención delimitan la absorción de escorrentías y el control de la inundación (Ka'zmierczak \& Cavan, 2011). Esta es una característica seleccionada del estudio en Great Manchester, que asocia una disfuncionalidad por el proceso de adaptación. La exposición se incrementa cuando las superficies en el sistema de drenaje no permiten la absorción de las escorrentías (Ka'zmierczak \& Cavan, 2011).

En el arroyo la Esmeralda, las aguas no pueden ser absorbidas por que el material de la canalización impermeabiliza el suelo aumentando el caudal; sin embargo, evita afectaciones en la vivienda cercana. Además, la baja cantidad de áreas verdes, la percepción de la comunidad sobre la función y su comportamiento negativo con la presencia, mantenimiento y limpieza restringe las posibilidades de permeabilidad (Arévalo,2016).

\section{Sistema de drenaje}

Según Ka'zmierczak \& Cavan, una característica física determinante en el incremento de la vulnerabilidad es el manejo inseguro e inadecuado de las escorrentías en la solución urbanística del asentamiento (2011) y en la medida estructural para mitigar el riesgo (Mustafa. 2009). La poca efectividad en el desagüe del terreno expone a la comunidad ante inundaciones.
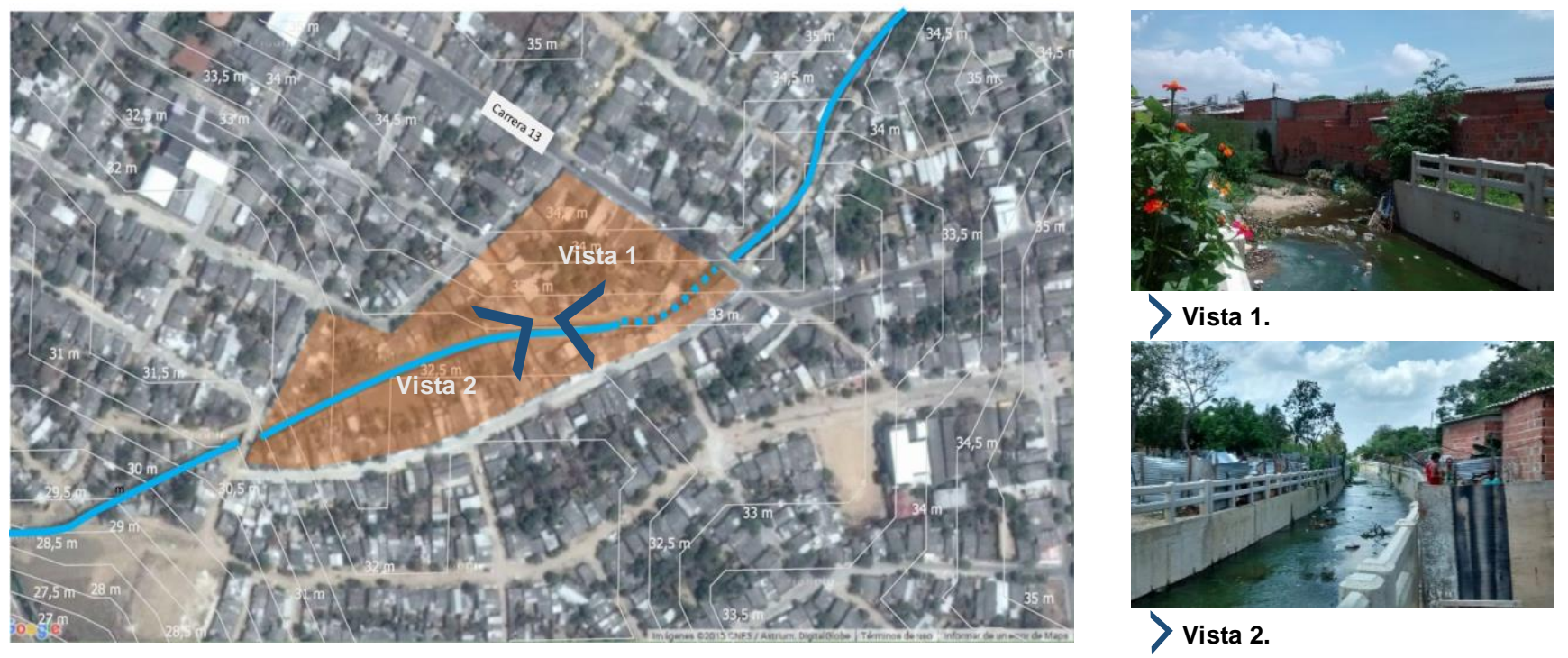

Gráfica 6. Sistema de drenaje en el caso de estudio.

Elaboración propia.

Refiere a una disfuncionalidad por el proceso de adaptación. Aunque en el sector Suroccidental se respeta el cauce natural del arroyo, estos se encuentran canalizados a cielo abierto, sus rondas hídricas han sido ocupadas por viviendas y en el entorno no se dispone de infraestructura de recolección y vertimiento de aguas lluvias (Ávila, 2015); las escorrentías circulan por la vía y alimentan el cauce del arroyo, ingresan a la vivienda y en algunos casos se estanca por las por obstáculos en la canalización. 


\section{Espacio público y Equipamiento}

Tanto espacio público como equipamiento tienen dos influencias en el comportamiento de la comunidad durante y después del momento de la inundación. Ayudan a desempeñar adecuadamente la protección conforme se dispongan en sitios seguros y la comunidad apropie el conocimiento sobre su función como refugio (Escuela de Hábitat, 2009). Además, los daños ocurridos en ambos perjudican las actividades colectivas. Al respecto, del sector de estudio, se manifiesta de forma negativa por su baja presencia y el desconocimiento de la comunidad de sitios de refugio o puntos seguros (Arévalo, 2016).

\subsection{Definición de las características de la vivienda propuestas}

\section{Patrón de exposición}

El patrón de exposición fue una característica intrínseca del caso de estudio detectada por el expediente urbano y la percepción de la comunidad. Muestra la localización de la vivienda respecto al sistema drenaje y morfología urbana como una disfuncionalidad formada en el proceso de adaptación al lugar; dependiendo de la localización de la vivienda, el emplazamiento en el lote, la tipología, la distancia con el arroyo y la relación entre morfología / sistema de drenaje se permite que la vivienda sea una estación para el desagüe de escorrentías (Arévalo, 2016).

En el sector estudiado del arroyo La Esmeralda, el 50\% de las viviendas se localizan sobre la zona de inundación del arroyo y presentan una relación directa con el desde su parte posterior o patio sin construcción de barreras, siendo el espacio de la casa más afectado. Por otra parte, se observó que la escorrentía de las vías aledañas entra por la vivienda hacia el arroyo. En menor medida, 12\%, de las viviendas presentan otro patrón que incide en espacios laterales o frontales (Ver gráfica 7) (Arévalo, 2016).

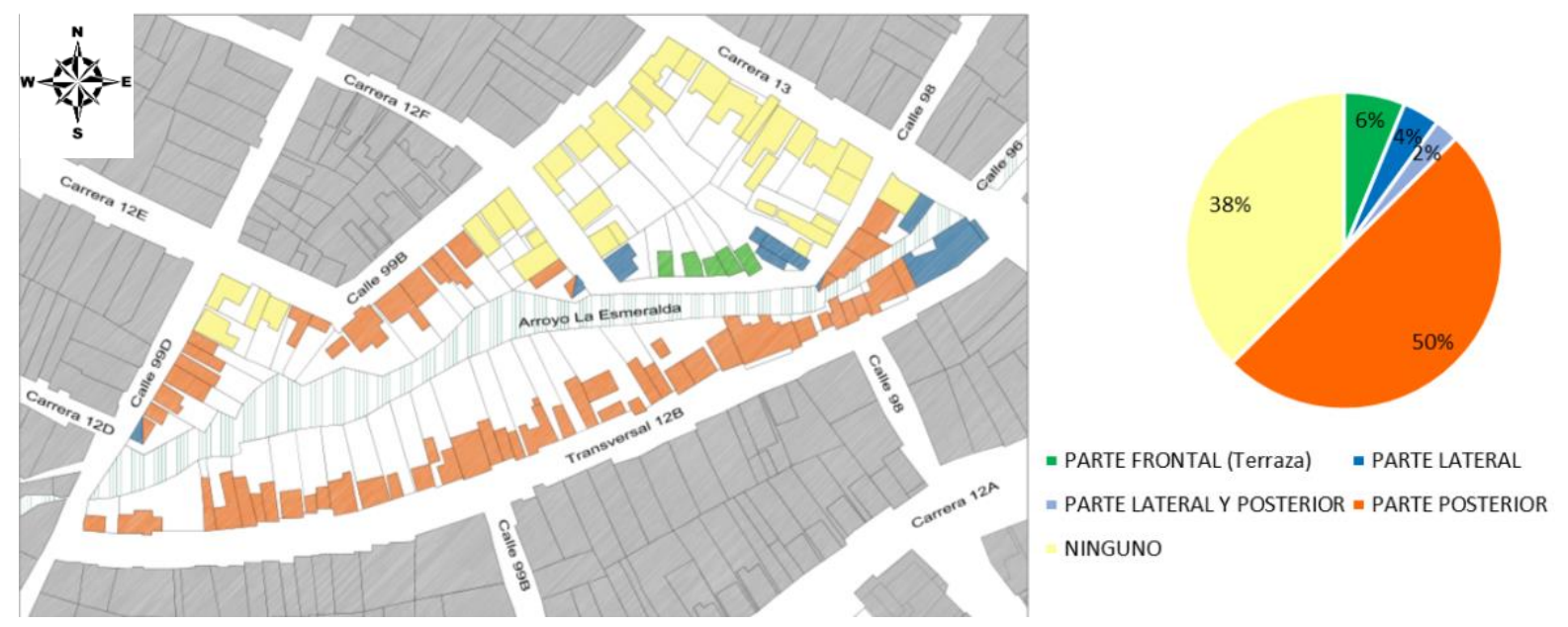

Gráfica 7. Patrón de exposición de la vivienda en el caso de estudio.

Elaboración propia para el expediente urbano.

\section{Tipología de vivienda}

La tipología de la vivienda (Ka'zmierczak \& Cavan, 2011) se reconoce del estudio de caso en Great Manchester, refiere a las características espaciales definidas en el proceso de autoconstrucción de esta, es decir, a la dimensión de disfuncionalidad por la forma de adaptación. Esta es la escala menor del territorio que mayor afectación presenta pues de acuerdo ellas se manifiesta la afectación por la inundación en el desarrollo adecuado de las actividades cotidianas de la familia y se debilita en su materialidad, estructura, muebles, enseres y electrodomésticos. De esa forma, permite acotar cómo es la exposición de la vivienda. Con el número de niveles (incluso con piso bajo o por debajo del nivel de la vía o anden) y tipología edificatoria, sea adosada, pareada o aislada (Arévalo,2016), correlacionados con la localización de la vivienda respecto al sistema de drenaje se conocen los espacios y los usos que se merman con la frecuencia de la inundación. Mientras que, el estado de la construcción y la materialidad ayuda a detectar los tipos de afectación en la vivienda (Escuela de Hábitat, 2009).

En el caso de estudio, con la comunidad, se verificaron las características de la vivienda que afectaban desde este aspecto tal como su tipología edificatoria. Se encontró que un $48 \%$ de las viviendas pertenece a una tipología continua (adosada en sus dos fachadas laterales al predio contiguo) y un $42 \%$ adosada de una fachada, indica 
que la mayoría de las viviendas pueden presentar una afectación a espacio internos por las escorrentías procedentes de vías aledañas que buscan salida al arroyo dado el patrón de exposición predominante (Arévalo, 2016).

\section{Conexión a servicios públicos}

Identificada en el estudio del Valle de Aburrá, señala la practicas de habitar nocivas para los hogares y los vecinos. Depende de la cobertura de servicios públicos básicos (acueducto, alcantarillado, luz y gas) que el Ente territorial ofrece y la posibilidad de conexión a esta. De acuerdo con el tipo de conexión, formal o informal, se incide en la calidad de vida de los hogares, pues no se puede garantizar el manejo de aguas servidas y evitar su vertimiento en el arroyo (Escuela de Hábitat, 2009). En el sector, aunque existe la disponibilidad de redes, las acometidas son informales (Alcaldía Distrital de Barranquilla, 2014).

\section{Manejo de aguas Iluvias en cubiertas y zonas exteriores}

La inundación está asociada a la magnitud, tiempo de lluvias y a las características del sistema de drenaje. Sin embargo, el manejo de las aguas en las superficies externas y de cubierta en la vivienda contribuye al caudal del arroyo, si el desagüe se realiza en el arroyo o en la vía. Las respuestas que los habitantes proporcionaron fueron encasilladas en el proceso de adaptación y las prácticas de habitar, donde se define el tipo de desagüe en patios, terrazas y cubierta. Para el caso de estudio se encontró que la mayoría de las viviendas tienen un manejo inadecuado de los desagües en estas superficies (ver gráfica 8) (Arévalo, 2016).
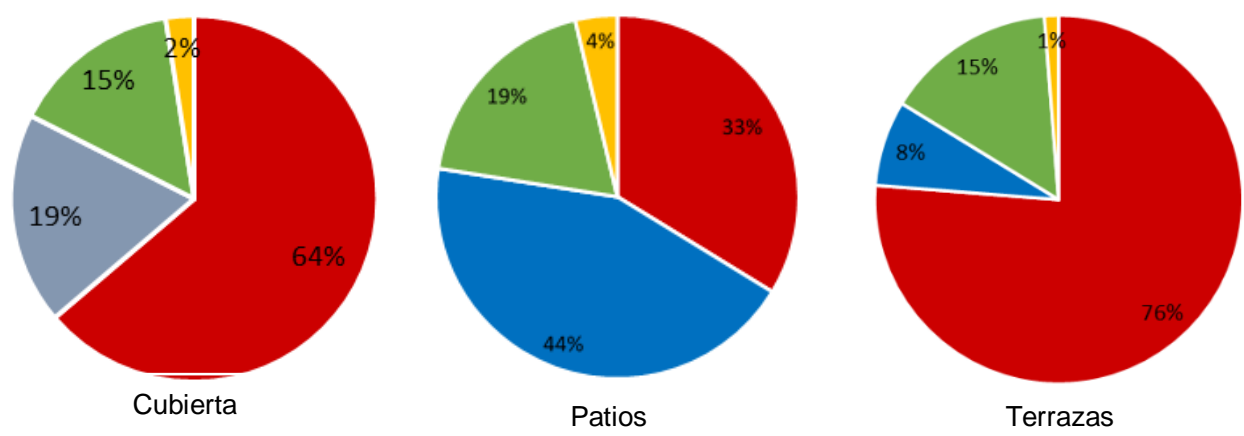

$$
\begin{aligned}
& \text { - Desagua hacia la calle } \text { - Desaguan hacia el arroyo } \\
& \text { n No sabe / No responde } \quad \text { Se envían al alcantarillado }
\end{aligned}
$$

Gráfica 8. Manejo de aguas Iluvias en Cubiertas, Patios y Terrazas.

Elaboración propia para el expediente urbano

\section{Medidas de autoprotección}

Las medidas demuestran la capacidad de prepararse de los habitantes pues se condicionan por el proceso de adaptación al lugar como respuesta al riesgo que enfrentan. De esa forma, se presentarán modificaciones en elementos arquitectónicos para protegerse de la inundación acorde a las limitaciones de ingreso económico del hogar. Alguna de estas, que se pueden observar en el caso de estudio, van desde elevar el nivel de la vivienda respecto a la vía, sea en espacios internos o externos y construir muros con alturas de $20 \mathrm{~cm}$ o $50 \mathrm{~cm}$ en los accesos (Arévalo, 2016).

Las características encontradas desde la micro escala delimitan una situación inherente en el sector Suroccidental que actúan en la magnitud de la vulnerabilidad social, informan acerca de situaciones ignoradas para la toma de decisiones. Según la Escuela de Hábitat, la estructura de la ciudad y las condiciones urbanísticas generadas en el proceso de adaptación influyen en la capacidad de respuesta (2009). Del mismo modo, desde la escala de vivienda, su tipología, la forma de conexión a redes de servicios públicos, el patrón de exposición, el manejo de las aguas lluvias en cubierta y zonas exteriores son características que influyen en el incremento de las condiciones de exposición. Otras como las medidas de intervención asumidas por los habitantes pueden limitar la respuesta al momento del desastre (Arévalo, 2016). Se destacan características que son propias del caso de estudio que no fueron vinculadas en la metodología aplicada para el instrumento de planificación del Distrito. 


\section{CARACTERÍSTICAS URBANAS Y LA VIVIENDA EN LA METODOLOGÍA DE EVALUACIÓN DE LA VULNERABILIDAD REALIZADA POR EL DISTRITO DE BARRANQUILLA}

En la medición que se incorporó al Plan de Ordenamiento Territorial, se verificaron características materiales y estructurales de la vivienda y la exposición de elementos urbanos, ambos medidos por la cantidad probable de ser afectada o dañada por la inundación. Ese enfoque no representa la complejidad sistémica que se configura en el hábitat vulnerable en el Suroccidente, de acuerdo con su origen y modos de habitar. En su lugar, las características son medidas como elementos aislados que luego se asumen en una sumatoria de elementos por escala. La complejidad manifiesta en el concepto del hábitat permite reconocer las situaciones simultaneas que se enfrentan.

En esta propuesta se develan, desde la dimensión físico-espacial, aquellas que pueden estar influenciando la magnitud de la vulnerabilidad. A escala de vivienda, se establece que la tipología edificatoria y el patrón de exposición inducen a mayores daños al enfrentar la inundación pues define las afectaciones parciales o totales en su espacialidad, mientras que, el comportamiento de la inundación es condicionado por el manejo de aguas lluvias y las medidas de autoprotección.

\begin{tabular}{|c|c|c|c|}
\hline & \multicolumn{3}{|c|}{ CARACTERISTICAS FISICO ESPACIALES } \\
\hline & Consideradas desde la comunidad cientifica & $\begin{array}{l}\text { Aplicadas en el instrumento de } \\
\text { planificación }\end{array}$ & Propuestas en este articulo \\
\hline \multirow{10}{*}{ VIVIENDA } & Stock de la vivienda y su tenencia & & \\
\hline & Tipologia de la vivienda & & Tipologia de la vivienda \\
\hline & Localización respecto a la fuente del riesgo & & \\
\hline & Aspectos estructurales de la vivienda & Altura de la edificación & \\
\hline & Materiales predominantes de la vivienda & $\begin{array}{c}\text { Caracteristicas de materiales de la } \\
\text { vivienda }\end{array}$ & \\
\hline & Conexión a servicios públicos de la vivienda & & Conexión a Servicios públicos de la vivienda \\
\hline & Estado de deterioro de la vivienda & Estado de deterioro de la edificación & \\
\hline & & & Patrón de exposición \\
\hline & & & $\begin{array}{c}\text { Manejo de aguas lluvias en superficies } \\
\text { exteriores de la vivienda }\end{array}$ \\
\hline & & & Medidas de autoprotección en la vivienda \\
\hline \multirow{5}{*}{$\begin{array}{l}\text { ENTORNO } \\
\text { INMEDIATO }\end{array}$} & Proporción de espacio verde & & Proporción de espacio verde \\
\hline & Disposición de aguas residuales & & \\
\hline & Disposición de residuos sólidos & & \\
\hline & Espacio público y equipamiento & Área de espacio Público expuesto & Espacio público y equipamiento \\
\hline & Sistema de drenaje pluvial & & Sistema de drenaje pluvial \\
\hline \multirow{5}{*}{$\begin{array}{l}\text { SECTORI } \\
\text { CIUDAD }\end{array}$} & Accesibilidad y conectividad & Longitud de vias expuestas & Accesibilidad y conectividad \\
\hline & Redes de servicios públicos & $\begin{array}{l}\text { Longitud red acueducto / alcantarillado } \\
\text { expuesto }\end{array}$ & Redes de servicios públicos \\
\hline & Densidad del entorno construido & & \\
\hline & Categorias de uso del suelo & Áreas de usos de suelo expuesto & \\
\hline & Densidad de la vivienda & & \\
\hline
\end{tabular}

Gráfica 9. Tabla comparativa entre las características físico-espaciales aplicadas en el instrumento de planificación y la propuesta. Elaboración propia.

Con ello, no indica omitir las afectaciones por el estado físico, el tipo de materiales empleados en la construcción y la altura de la vivienda, medidas en por el instrumento de planificación Distrital, solo se establece que no son suficientes para medir una situación que incide principalmente con afectaciones al modo de vida y la estructura de ingreso/gastos de los hogares. Siempre y cuando se establezca la calidad del diseño y los materiales empleados junto con la confiabilidad de las técnicas de construcción empleadas y la formación técnica de los actores que intervienen (Escuela de Hábitat, 2009). Admitiendo una visión completa sobre el origen del estado de la vivienda. En otras escalas, además de reconocer la cantidad de vías, redes de servicio público, usos de suelo y equipamiento urbano expuesto, el análisis puede ser complementado con la identificación de sitios de refugio o rutas de evacuación seguras que integra la capacidad de respuesta de la comunidad.

Cada elemento físico desde las distintas escalas aporta e influencian la vulnerabilidad y su comprensión se logra si se estudian las diversas relaciones y su calidad al cambiar el enfoque de la vulnerabilidad como un estado inherente (Cutter, 2008) del hábitat en un lugar con características no homogéneas. 


\section{VARIABLES}

Las variables asumidas en el estudio para la incorporación al instrumento de planificación de Barranquilla fueron Exposición, fragilidad (social y física) y capacidad de respuesta de las personas, medidas a partir de una sumatoria de circunstancias que las observa de forma aislada. En su lugar, en esta propuesta, se considera la importancia del enfoque sistémico en el concepto del hábitat residencial del INVI: "es el resultado de un proceso en permanente conformación de lugares en distintas escalas referidas al territorio, que se distinguen por una forma particular de apropiación, dado por un vínculo cotidiano con unidades de experiencias singulares, potenciando relaciones de identidad y pertenencia, a partir de lo cual el habitante lo interviene y configura" (2011), fiel a las dinámicas que se manifiestan en entornos vulnerables y que influyen en la mayor o menor medida de la vulnerabilidad.

De acuerdo con el interés de indicar aquellas características físicas de los asentamientos informales que condicionan la vulnerabilidad para sistematizar unas variables de análisis, se plantea que la base es reconocer que "la dinámica está enmarcada en unas condiciones de sensibilidad cambiante de la comunidad a los impactos de la inundación dentro de un entorno físico estático." (Arevalo,2016:135). Resultan de ese enfoque cuatro variables para aportar en el conocimiento de la vulnerabilidad:

\section{a. Calidad de las relaciones:}

Todas las relaciones prejudiciales son resultados de la interacción entre la vivienda, el sistema de drenaje, el entorno construido, además, se vincula las relaciones entre ellas, como filtro físico (Cutter et. al., 2003), y la comunidad. La complejidad del hábitat y sus relaciones sistémicas son disfuncionales, dos tipos de relaciones pueden medir la calidad y su manifestación en la vulnerabilidad:

- Relaciones espaciales entre los elementos naturales y construidos en un entorno de amenaza por inundaciones. Son aquellas relaciones que incrementan las condiciones de exposición y permiten la magnitud de la inundación. De acuerdo con la relación entre vivienda-arroyo, vivienda-manzana y manzana-entorno inmediato se puede conocer cómo es la exposición en referencia con las características tipológicas de la vivienda, localización, patrón de exposición, sistema de drenaje y la proporción de espacios verdes o permeables (Arévalo, 2016).

- Relaciones socio-espaciales, Refiere a las relaciones espaciales, a escala de sector-ciudad, que la comunidad asume dentro de su capacidad de respuesta. La presencia de equipamientos y espacio público, accesibilidad y conectividad junto con el reconocimiento como sitios de refugio y rutas de evacuación, construyen una respuesta rápida y segura (Arévalo, 2016).

\section{b. Exposición bajo condiciones resultada del proceso de construcción y adaptación del lugar:}

Es una variable que ayuda a identificar los daños futuros en la cotidianidad de los habitantes. La vivienda es el soporte físico del hábitat, se asume que las condiciones estructurales, materiales soportadas en la confiabilidad técnica de construcción y la percepción sobre el estado de riesgo para tomar decisiones de intervención son componentes que influyen en incrementar la exposición (Escuela de Hábitat, 2009).

c. Prácticas de Habitar. En esta variable la visión de la Escuela de Hábitat en el Valle de Aburrá (2009) y la observación en el caso de estudio fue definitiva para proponer que existe una influencia de las prácticas y actividades cotidianas que la comunidad desarrolla para protegerse de los impactos de la inundación, tales como intervenciones en medidas de protección y manejo de las aguas en superficies externas de la vivienda (Arévalo, 2016). Estas son intervenciones poco significativas para la exigencia de protección ante el impacto y dependen de la capacidad de la comunidad para implementarlas. 


\begin{tabular}{|c|c|c|c|}
\hline \multirow{2}{*}{$\begin{array}{l}\text { VARIABLES } \\
\text { PROPUESTAS }\end{array}$} & \multicolumn{3}{|c|}{ CARACTERISTICAS PROPUESTAS } \\
\hline & VIVIENDA & ENTORNO INMEDIATO & SECTOR/CIUDAD \\
\hline \multirow{2}{*}{$\begin{array}{l}\text { RELACIONES } \\
\text { ESPACIALES }\end{array}$} & Tipología de la vivienda & $\begin{array}{c}\text { Proporción de espacio } \\
\text { verde }\end{array}$ & \\
\hline & Patrón de exposición & Sistema de drenaje & \\
\hline $\begin{array}{l}\text { RELACIONES } \\
\text { SOCIO- } \\
\text { ESPACIALES } \\
\end{array}$ & & $\begin{array}{l}\text { Espacio público y } \\
\text { equipamiento }\end{array}$ & $\begin{array}{l}\text { Accesibilidad y } \\
\text { conectividad }\end{array}$ \\
\hline \multirow[t]{2}{*}{$\begin{array}{l}\text { EXPOSICIÓN POR } \\
\text { PROCESO DE } \\
\text { CONSTRUCCIÓN } \\
\text { Y ADAPTACIÓN } \\
\text { DEL LUGAR }\end{array}$} & $\begin{array}{c}\text { Características } \\
\text { espaciales y materiales } \\
\text { (Materiales de } \\
\text { construcción, aspecto } \\
\text { estructural y altura de la } \\
\text { edificación) }\end{array}$ & & \\
\hline & $\begin{array}{l}\text { Estado de deterioro de } \\
\text { la edificación }\end{array}$ & & \\
\hline \multirow{3}{*}{$\begin{array}{l}\text { PRACTICAS DE } \\
\text { HABITAR }\end{array}$} & $\begin{array}{l}\text { Conexión a servicios } \\
\text { públicos }\end{array}$ & & $\begin{array}{c}\text { Redes de Servicios } \\
\text { Públicos. }\end{array}$ \\
\hline & $\begin{array}{l}\text { Medidas de } \\
\text { autoprotección }\end{array}$ & & \\
\hline & $\begin{array}{c}\text { Manejo de las aguas } \\
\text { Iluvias en cubiertas, } \\
\text { patios y terrazas de la } \\
\text { vivienda }\end{array}$ & & \\
\hline
\end{tabular}

Gráfica 10. Relación entre variables y características propuestas para el estudio de la vulnerabilidad en el área de estudio del Arroyo La Esmeralda en la ciudad de Barranquilla.

Elaboración propia.

\section{CONCLUSIONES}

Este articulo indica que las relaciones en el hábitat vulnerable son disfuncionales porque asume la exposición como una condición que no depende únicamente de la localización en una zona de amenaza, los cambios en el entorno físico al ser poco significativos para superar el estado y las medidas de protección propios de la comunidad se conjugan en mayor o menor medida por las características de la vivienda enfrentadas a unas características del entorno. Esa interacción de elementos influencia cada hogar y lo hace sensible al comportamiento de la inundación; así las medidas de mitigación se podrían dirigir a corregir más aspectos del estado y no enfocarse solo en la canalización del arroyo.

Los recursos invertidos por la administración pública serían más efectivos si se concentran en la comprensión sistémica y escalar de la vulnerabilidad que no es homogénea, como se enfatiza en el instrumento de planificación. El crecimiento urbano en el sector suroccidental de la ciudad, como filtro físico, es diverso y la morfología resultada tiene influencia en el comportamiento de la inundación, por lo tanto, características físicas de la vivienda y entorno inmediato incrementan la exposición y estarían limitando la capacidad de respuesta al ser diversas las situaciones que se enfrentan.

La operacionalización manejada para la investigación presentada permite estudiar un concepto de vulnerabilidad abstracto y analizado desde una perspectiva cualitativa que trasciende la identificación de elementos expuestos y llega a comprender el funcionamiento del hábitat en condiciones de vulnerabilidad. Ese reconocimiento se asume como una red de relaciones entre elementos, donde la exposición es la interacción que configura la magnitud del estado; por lo tanto los impactos no se pueden evidenciar únicamente en daños materiales y económicos o en una capacidad de respuesta limitada solo por la estructura de ingresos/gastos de los hogares, cuando los impactos también son influyentes en el modo de vida, el correcto funcionamiento de las actividades cotidianas y las afectaciones en las dimensiones sicológicas, entendiendo por estas estado emocional y expectativas de superación del estado. De esta forma, la condición de vulnerabilidad es inherente y es delimitada por las características de cada lugar (Cutter, 2008). Además, estas relaciones entre la vivienda y el entorno inmediato también se desarrollan con elementos que corresponden a las distintas escalas territoriales. 
En las características del instrumento de planificación, analiza características similares pero el enfoque de medición no permite establecer claramente su incidencia en el estado de vulnerabilidad, por la escala de estudio. El análisis desde la micro escala muestra las diferentes situaciones que enfrentan los habitantes; es una información que expone diversas situaciones físicas posibles de incrementar o no la vulnerabilidad social. Se puede deducir acerca de la distribución, al ser abordada desde el análisis en el sector y llegar a diferenciar otros niveles de vulnerabilidad, incluso más inherentes o particulares para el Distrito.

\section{BIBLIOGRAFÍA}

ALCALDÍA DISTRITAL DE BARRANQUILLA (2014) Documento de Soporte Técnico Plan de Ordenamiento Territorial del Distrito de Barranquilla. Barranquilla. http://www.barranquilla.gov.co/normatividad/leyes-yacuerdos/cat view/226-nuevo-plan-de-ordenamiento-territorial/250- (Consulta: 14/02/2014)

ARÉVALO, M. (2016). Asentamientos informales, inundaciones y vulnerabilidad fisico-espacial de la vivienda y el entorno inmediato. Caso de estudio arroyo La Esmeralda en la ciudad de Barranquilla, Colombia. http://repositorio.uchile.cl/handle/2250/140635 (Consulta: 25/01/2018)

ÁVILA, H. (2015). Perspectiva del manejo del drenaje pluvial frente al cambio climático - caso de estudio: ciudad de Barranquilla, Colombia. Revista de Ingeniería Universidad de los Andes, 36, 54-59. http://www.scielo.org.co/pdf/ring/n36/n36a11.pdf (Consulta 15/01/2018)

BANCO MUNDIAL. (2012). Análisis de la gestión del riesgo de desastres en Colombia: un aporte para construcción de políticas públicas. Bogotá: Editorial Banco Mundial. http://www.sigpad.gov.co/sigpad/archivos/GESTIONDELRIESGOWEB.pdf (Consulta: 15/06/2013)

BIRKMANN, J. (2006). Indicator and criteria for measuring vulnerability: Theoretical bases and requirements. En J. BIRKMANN, Measuring Vulnerability to Natural Hazards: Towards Disaster Resilient Societies (págs. 55-77). India: United Nations University Press.

ESCUELA DEL HÁBITAT UNIVERSIDAD NACIONAL DE COLOMBIA, E. D. (2009). Informe Final Convenio 4800002397 de $2007 . \quad$ Medellín. http://www.metropol.gov.co/Planeacion/DocumentosAreaPlanificada/Informe\%20III_Vulnerabilidad.pdf (Consulta: 11/01/2015)

CUTTER, S., et. al. (2008). A place-based model for understanding community resilience. GlobalmEnviromental Change, 18(Issu 4), 598-606. http://www.sciencedirect.com/science/article/pii/S0959378008000666 (Consulta: 14/03/2012)

CUTTER, S., BORUFF, B., \& SHILEY, W. (2003). Social Vulnerebility to Environmental Hazards. Social Science Quarterly, 84(1), 242-261. http://onlinelibrary.wiley.com/wol1/doi/10.1111/1540-6237.8402002/full (Consulta: 06/09/2012)

KA'ZMIERCZAK, A., \& CAVAN, G. (2011). Surface water flooding risk to urban communities: Analysis of vulnerability, hazard and exposure . Landscape and Urban Planning, 103(2), 185-197. http://www.sciencedirect.com/science/article/pii/S0169204611002404 (Consulta: 17/05/2013)

LINDLEY, S., O'NEILL, J., KANDEH, J., LAWSON, N., CHRISTIAN, R., \& O'NEILL, M. (2011). Climate Change, justice and vulnerability. Joseph Rowntree Foundation. https://www.jrf.org.uk/report/climate-changejustice-and-vulnerability (Consulta: 03/03/2015)

LOMBARD, M. (2015). Lugarización y la construcción de asentamientos informales en México. Revista INVI, 30(83), 79-115. http://revistainvi.uchile.cl/index.php/INVI/article/view/911 (Consulta: 05/05/2015)

MUSTAFA, D. et al. (2011). Pinning down vulnerability: from narratives to numbers. Disasters, 35(1), 62-86. http://onlinelibrary.wiley.com/doi/10.1111/j.1467-

7717.2010.01193.x/abstract;jsessionid=3341983ABED727070B12CE9786B6A585.f01t02 (Consulta: $14 / 03 / 2014)$

PÉREZ, S. (2014). La pobreza en emergencia: Nuevas dimensiones en situaciones de crisis. En BOLVINIK, Multidimensionalidad de la pobreza. Propuestas para su definición y evaluación en América Latina y el Caribe (457-486). Buenos Aires: CLACSO. http://biblioteca.clacso.edu.ar/clacso/clacsocrop/20131204115049/Multidimensionalidad.pdf (Consulta: 20/07/2015)

ROMERO, H., \& MENDOCA, M. (2012). Amenazas naturales y evaluación subjetiva en la construcción de la vulnerabilidad social ante desastres naturales en Chile y Brasil. Intethesis, Florianópolis, 9(1), 127-180. https://www.rbcdh.ufsc.br/index.php/interthesis/article/download/1807-1384.2012v9n1p127/22509

(Consulta: 23/04/2015) 